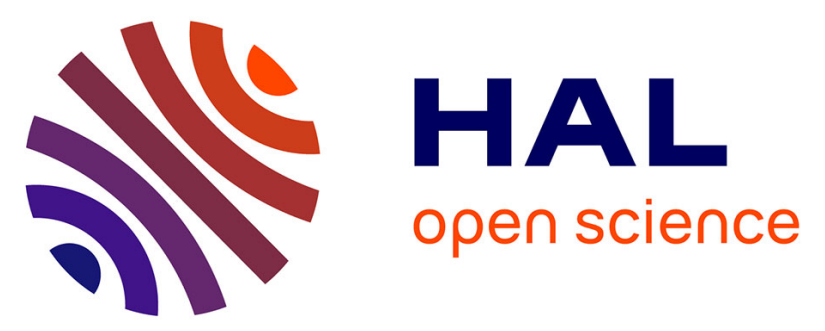

\title{
Benzophenone Photoreactivity in a Lipid Bilayer To Probe Peptide/Membrane Interactions: Simple System, Complex Information
}

\author{
Leïla Bechtella, Carla Kirschbaum, Marine Cosset, Gilles Clodic, Lucrèce \\ Mathéron, Gérard Bolbach, Sandrine Sagan, Astrid Walrant, Emmanuelle \\ Sachon
}

\section{To cite this version:}

Leïla Bechtella, Carla Kirschbaum, Marine Cosset, Gilles Clodic, Lucrèce Mathéron, et al.. Benzophenone Photoreactivity in a Lipid Bilayer To Probe Peptide/Membrane Interactions: Simple System, Complex Information. Analytical Chemistry, 2019, 91 (14), pp.9102-9110. 10.1021/acs.analchem.9b01584 . hal-02346638

\section{HAL Id: hal-02346638 \\ https://hal.science/hal-02346638}

Submitted on 15 Oct 2020

HAL is a multi-disciplinary open access archive for the deposit and dissemination of scientific research documents, whether they are published or not. The documents may come from teaching and research institutions in France or abroad, or from public or private research centers.
L'archive ouverte pluridisciplinaire HAL, est destinée au dépôt et à la diffusion de documents scientifiques de niveau recherche, publiés ou non, émanant des établissements d'enseignement et de recherche français ou étrangers, des laboratoires publics ou privés. 


\title{
Benzophenone photoreactivity in a lipid bilayer to probe peptide/membrane interactions: simple system, complex information
}

\author{
Leïla Bechtella, ${ }^{\mathrm{a}}$ Carla Kirschbaum, ${ }^{\mathrm{a}, \mathrm{b}}$ Marine Cosset, ${ }^{\mathrm{a}}$ Gilles Clodic, ${ }^{\mathrm{b}}$ Lucrèce Matheron, ${ }^{\mathrm{b}}$ Gérard \\ Bolbach, ${ }^{\mathrm{a}, \mathrm{b}}$ Sandrine Sagan, ${ }^{\mathrm{a}}$ Astrid Walrant, ${ }^{\mathrm{a}}$ Emmanuelle Sachon*a,b
}

aSorbonne Université, École normale supérieure, PSL University, CNRS, Laboratoire des Biomolécules, LBM, 75005 Paris, France.

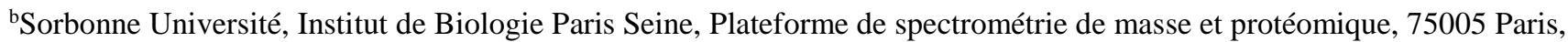
France

* emmanuelle.sachon@ sorbonne-universite.fr

Benzophenone photoreactivity $\bullet$ Lipids $\bullet$ Mass spectrometry $\bullet$ Photoaffinity labelling $\bullet$ Cell-penetrating peptides

\begin{abstract}
Affinity photocrosslinking coupled to mass spectrometry, using benzophenone (Bzp)-functionalized peptides, was used to study the non-covalent interactions of cell-penetrating peptides and lipid membranes. Using biomimetic lipid vesicles composed of saturated and unsaturated negatively charged lipids: DMPG (14:0), DPPG (16:0), DOPG (18:1 cis $\left.\Delta^{9}\right)$, $18: 1$ (trans $\left.\Delta^{9}\right)$ PG and DLoPG $\left(18: 2\right.$ cis $\left.\Delta^{9,12}\right)$, allowed to observe all the classical and less common reactivities of Bzp described in the literature by direct MS analysis: $\mathrm{C}=\mathrm{C}$ double bond formation on saturated fatty acids, covalent adducts formation via classical $\mathrm{C}-\mathrm{C}$ bond and Paternò-Büchi oxetane formation followed or not by fragmentation (retro-Paternò-Büchi) as well as photosensitisation of unsaturated lipids leading to lipid dimers. All these reactions can occur concomitantly in a single complex biological system: a membrane-active peptide inserted within a phospholipid bilayer. We also detect oxidation species due to the presence of radical oxygen species. This work represents a noteworthy improvement for the characterisation of interacting partners using Bzp photocrosslinking, and shows how to exploit in an original way the different reactivities of Bzp in the context of a lipid membrane. We propose an analytical workflow for the interpretation of MS spectra, giving access to information on the CPP/lipid interaction at a molecular level such as depth of insertion or membrane fluidity in the CPP vicinity. An application of this workflow illustrates the role of cholesterol in the CPP/lipids interaction.
\end{abstract}

\section{INTRODUCTION}

Benzophenone (Bzp) is a photoprobe with exceptionally diverse photoreactivity and fields of use. ${ }^{1}$ In particular, it is a photocrosslinking reagent of choice for the study of biomolecular non-covalent interactions. It can be easily coupled to biomolecules, it is biocompatible and its triplet biradical excited state is in equilibrium with the ground state, which provides a certain photostability of labelled molecules, ${ }^{1}$ allowing their use over the long incubation times often needed when studying biological systems. The reactivity of the $n-\pi^{*}$ triplet state (Figure 1 , molecule $1^{*}$ ) is extremely well characterised. When used as a photocrosslinking reagent, $\mathrm{H}^{*}$ abstraction type reactivity is exploited to create a covalent $\mathrm{C}-\mathrm{C}$ bond between two non-covalent interaction partners. This reaction is shown on Figure 1 in the specific context of a photoreactive Bzp-modified peptide and a lipid interaction partner ( $\mathrm{C}-\mathrm{C}$ bond, molecules 2 and 3). Covalent bond formation can also occur through a $[2+2]$ cycloaddition on a $\mathrm{C}=\mathrm{C}$ double bond leading to an oxetane ring (Paternò-Büchi reaction, $\mathrm{P}-\mathrm{B}$ ). This reaction is shown on Figure 1 (Oxetane, molecule 5). Other secondary reactions have also been described, such as dimerisation of molecules containing $\mathrm{C}=\mathrm{C}$ double bonds (Figure 1, lipid dimer, molecule 7) after sensitisation of the corresponding molecule by triplet excited Bzp (Figure 1, molecule 6*), or Bzp-assisted photooxidation through $\mathrm{H}^{*}$ abstraction leading to the formation of a new $\mathrm{C}=\mathrm{C}$ double bond (Figure 1, double bond, molecule 4). ${ }^{2,3}$ Most of these reactions are reported in the literature and were identified independently using small molecules in solution. ${ }^{4}$ In the present study, we used Bzp photocrosslinking coupled to mass spectrometry (MS) to study the non-covalent interactions between cell penetrating peptides (CPP) and lipid bilayers (Figure 2).

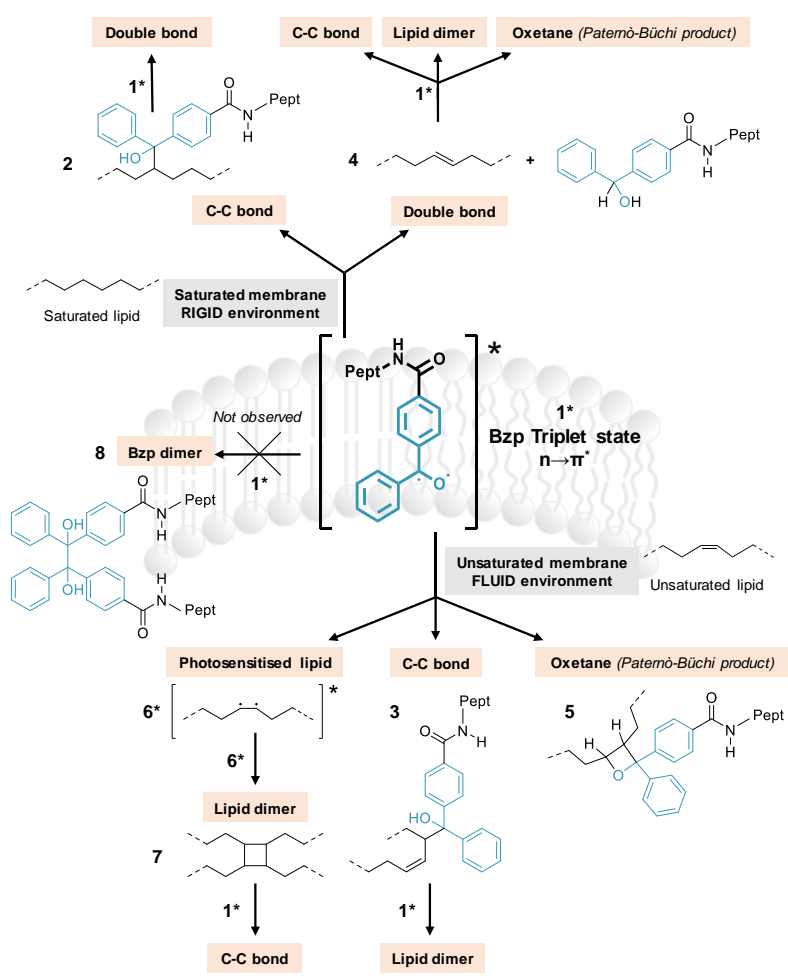

Figure 1. Observed Photochemical reactions of the Bzp triplet biradical $(1 *)$ in model membranes composed of saturated or unsaturated lipids (See text). 

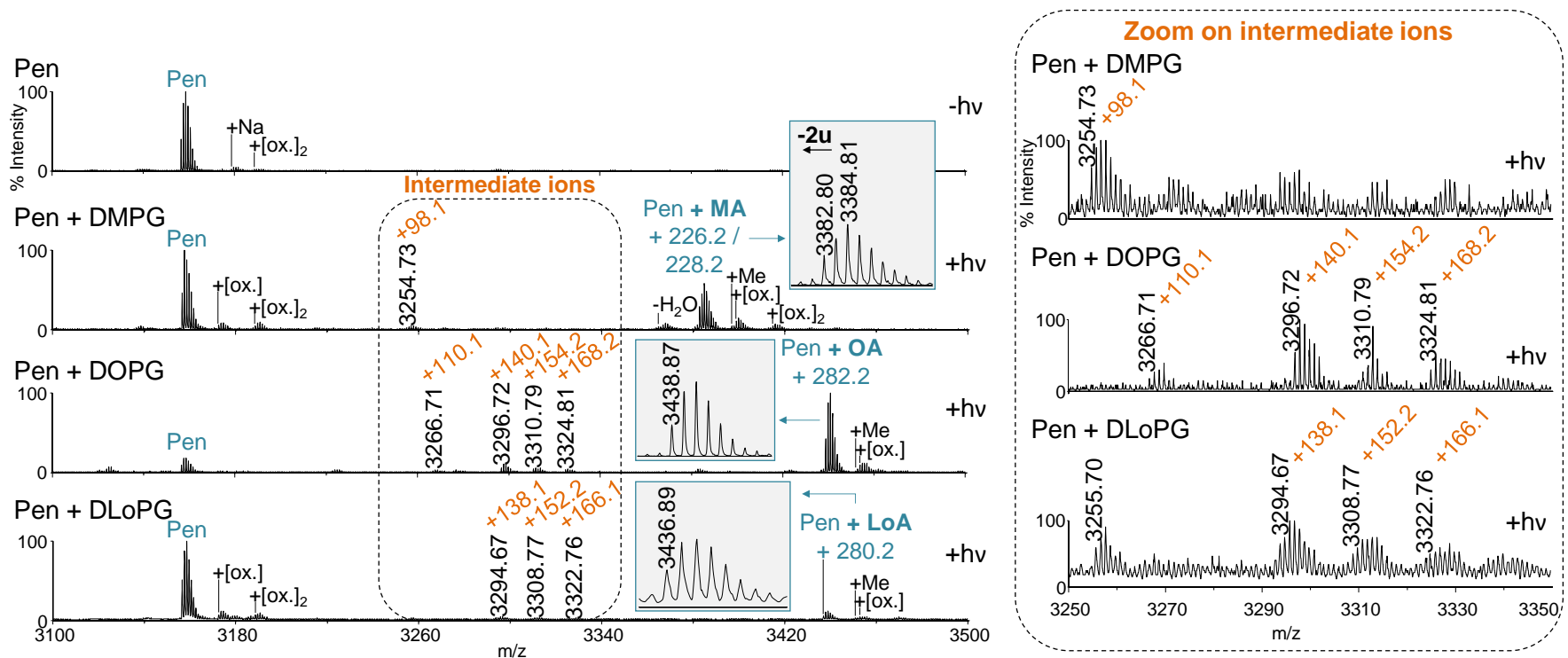

Figure 2. MALDI-TOF spectra (positive ions, reflector mode) of the Bzp-induced photocrosslinking between Pen and negatively charged Multilamellar Vesicles (MLVs) of various fatty acid chains. Top to bottom: Control Pen without irradiation (-hv), Pen+DMPG+hv, Pen+DOPG $+h v$, Pen+DLoPG $+h v$. Similar conditions of analysis were used in all spectra of the manuscript, 2,5-DHB is used as the matrix. Me: methylation, Na: sodium adduct, [ox.]: oxidation. The panel on the right is a zoom on the intermediate ions in the region $3250<\mathrm{m} / \mathrm{z}<3350$.

CPPs are a class of membrane-active peptides that can cross cell membranes and deliver biologically active molecules into cells. Understanding direct translocation of CPPs through lipid membranes is of prime interest for cytosol-targeted delivery. Direct translocation is a purely physico-chemical process involving membrane destabilisation by the CPP through interaction with the lipids of the bilayer. Getting a precise molecular knowledge of CPP/lipids interactions is crucial for a better understanding of those internalisation pathways. ${ }^{5}$

Photocrosslinking-based interactomics is a developing field, in particular for the study of protein or peptide-membrane interactions, using either photoreactive lipids, ${ }^{6-10}$ or photoreactive peptides or proteins. ${ }^{11}$ There are very few studies focusing on identification of CPPs membrane interaction partners. In 2016, the proteoglycan syndecan-4 was identified as a primary cell-surface target for octaarginine, using a diazirinemodified peptide. ${ }^{12}$ Work from our group focused on Bzpfunctionalised CPPs to identify lipid-type partners. ${ }^{13,14}$ Herein, we give a thorough mapping of the photoreactivity of Bzp coupled to two different CPPs (Penetratin and $(\mathrm{R} / \mathrm{W})_{9}{ }^{15}$ ) to characterise their interaction with biomimetic lipid membranes. This model allowed to observe all the classical and less common reactivities of Bzp described on Figure 1 by direct MS analysis. We show that all these reactions can occur concomitantly in a single complex biological system: a membrane-active peptide inserted within a phospholipid bilayer. In particular, we show how they compete depending on the nature of the phospholipids we used and thus of the physico-chemical state of the membrane surrounding Bzp. Analysis of all the described reaction products can significantly improve characterisation of CPP-membrane interactions using Bzp photocrosslinking coupled to MS, by providing at least three types of information. Identification of the classical $\mathrm{C}-\mathrm{C}$ bond adduct(s) will give information on the nature of the lipids in the immediate peptide environment. Unusual isotopic distribution for the adduct peak can be used as a marker of membrane fluidity around the peptide. Detection and identification of intermediate ions will provide information on the depth of insertion of the peptide in the membrane. All this information is obtained at the
MS level directly, thus limiting the amounts of material needed. After a comprehensive description and characterisation of all the side reaction products we observed, we will illustrate how to exploit them via a simple analytical workflow allowing direct MS spectra interpretation leading to information about interaction and insertion of this membrane-active peptide in lipid bilayer with binary mixtures. The final goal of this study is to understand how membrane permeability is influenced by a CPP sequence, how the lipids structure influences entry of CPP into cells and to locate preferred interaction sites between CPP and lipids.

\section{EXPERIMENTAL SECTION}

\section{Peptide synthesis.}

$\operatorname{Biot}\left(\mathrm{O}_{2}\right)-\mathrm{G}_{5}-\mathrm{K}(\varepsilon \mathrm{Bzp})-\mathrm{RQIKIWFQNRRM}\left(\mathrm{O}_{2}\right) \mathrm{KWKK} \mathrm{NH}_{2}$, $[\mathrm{M}+\mathrm{H}]^{+}, \quad \mathrm{m} / \mathrm{z} \quad 3156.62 \quad(\mathrm{Pen}), \quad \operatorname{Biot}\left(\mathrm{O}_{2}\right)-\mathrm{Apa}_{-} \mathrm{G}_{5}-\mathrm{K}(\varepsilon \mathrm{Bzp})-$ RQIKIWFQNRRM $\left(\mathrm{O}_{2}\right) \mathrm{KWKK} \mathrm{NH}_{2},[\mathrm{M}+\mathrm{H}]^{+}, \mathrm{m} / \mathrm{z} 3255.69$ with Apa: aminopentanoic acid (ApaPen) and $\operatorname{Biot}\left(\mathrm{O}_{2}\right)-\mathrm{G}_{5}-\mathrm{K}(\varepsilon \mathrm{Bzp})-$ RRWWRRWRR-NH $\mathrm{NH}_{2},[\mathrm{M}+\mathrm{H}]^{+}, \mathrm{m} / \mathrm{z} 2392.20\left((\mathrm{R} / \mathrm{W})_{9}\right)$, were synthesised by standard Boc solid-phase peptide synthesis.

\section{Liposomes preparation.}

Multilamellar vesicles (MLVs) at $2 \mathrm{mM}$ lipid were prepared according to the standard hydrated film method in PBS pH 7.4 using Dimyristoyl phosphatidylglycerol (DMPG), Dipalmitoyl phosphatidylglycerol (DPPG) (Genzyme, Switzerland), Dioleyl phosphatidylglycerol (DOPG), Dielaidoyl phosphatidylglycerol (18:1 (trans $\Delta^{9}$ ) PG), Dilinoleyl phosphatidylglycerol (DLoPG), cholesterol (Chol) (Avanti Polar Lipids, USA). $\mathrm{T}_{\mathrm{m}}(\mathrm{DMPG})=23^{\circ} \mathrm{C}$, $\mathrm{T}_{\mathrm{m}}(\mathrm{DPPG})=41^{\circ} \mathrm{C}, \quad \mathrm{T}_{\mathrm{m}}(\mathrm{DOPG})=-18^{\circ} \mathrm{C}, \quad \mathrm{T}_{\mathrm{m}}\left(18: 1\right.$ trans $\left.\Delta^{9}\right)$ $\mathrm{PG} \approx 12^{\circ} \mathrm{C}$ and $\mathrm{T}_{\mathrm{m}}(\mathrm{DLoPG}) \approx-57^{\circ} \mathrm{C}$ (Structures on Figure $\mathrm{S} 1$ ).

\section{Bzp-induced photocrosslinking.}

Photocrosslinking experiments were performed on samples containing $10 \mathrm{nmol}$ peptide and $100 \mathrm{nmol}$ lipid (MLVs) in PBS, $200 \mu \mathrm{L}$ total volume, as previously described. ${ }^{13}$ The published protocol was significantly optimised on the following points. (1) Fully oxidised peptides using $\operatorname{Biot}\left(\mathrm{O}_{2}\right)$ and $\operatorname{Met}\left(\mathrm{O}_{2}\right)$ were 
synthesised. (2) A narrow spectrum UV-lamp (6 W, VWR) was used. (3) Sample irradiation was performed under controlled agitation and temperature $\left(45^{\circ} \mathrm{C}\right)$ over $1 \mathrm{~h}$. (4) Saponification was performed over a short time (10 min) in $2 \mathrm{M} \mathrm{NaOH}$. Photoadducts were captured on streptavidin-coated magnetic beads. For MALDI-TOF analysis, samples were eluted with 2,5-DHB matrix $\left(1 \% \mathrm{H}_{3} \mathrm{PO}_{4}, \mathrm{pH} 1\right)$. For ESI analysis, samples were eluted with 20 $\% \mathrm{HCO}_{2} \mathrm{H}$, the recovery was less efficient than with 2,5-DHB.

\section{Mass spectrometry analysis.}

MALDI-TOF MS spectra were acquired on a 4700 Proteomics Analyzer (Applied Biosystems) or an Autoflex III (Bruker Daltonics), in positive ions reflector mode. MALDI-TOF acquisition parameters were optimised on Insulin chain B $(\mathrm{m} / \mathrm{z}$ 3494) for the detection of peptides around 3100-3500 u. For high energy CID $\left(\mathrm{N}_{2}\right.$ gas, $\left.1 \mathrm{keV}\right) \mathrm{MS} / \mathrm{MS}$ experiments (MALDI$\mathrm{TOF} / \mathrm{TOF}$ ), the precursor ion selection window was adjusted +/$7 \mathrm{u}$. For MALDI PSD experiments (Laser induced dissociation, post-source decay fragmentation), the analyser was operated in LIFT mode, the selection window was automatically chosen at $+/-$ $10 \%$ of the $\mathrm{m} / \mathrm{z}$ of the precursor ion.

For ESI flow-injection analysis (FIA), the solvent used was acetonitrile $/ \mathrm{H}_{2} \mathrm{O}(20 / 80)$ containing $0.1 \%$ formic acid, flow rate 30 $\mu \mathrm{L} / \mathrm{min}$. Spectra were acquired with a high resolution $(\mathrm{R}=100000)$ using the Orbitrap mass analyser (Thermo Scientifics).

\section{Studies and simulations of isotopic distributions.}

Theoretical isotopic distributions were calculated with the Isopro 3.0 software for the pure molecules. Theoretical distributions were shifted to the desired $\mathrm{m} / \mathrm{z}(-2 \mathrm{u})$. Simulations were obtained by combining this new distribution to the theoretical one, with the percentage of contribution of the two species. Contribution percentages were adjusted by comparing visually the simulations to the experimental isotopic distributions. Data treatment was performed with Excel.

\section{RESULTS AND DISCUSSION}

\section{General methodology and side reaction description}

Our previous study ${ }^{13}$ showed that Pen interacts preferably with negatively charged lipids so we chose to focus first on MLVs composed of PG phospholipids only for the full description of side reactions. In a second step we used binary mixtures of lipids: PG and Chol, to unveil Pen preferences according to bilayer fluidity properties. Photocrosslinking using the photoreactive and biotinylated Pen and the rationally designed CPP (R/W) ${ }_{9}$ was applied to MLVs composed of a single PG phospholipid and we varied the nature of the fatty acid chains. We used saturated DMPG (14:0) and DPPG (16:0), mono-unsaturated DOPG (18:1 cis $\left.\Delta^{9}\right)$ and 18:1 (trans $\left.\Delta^{9}\right)$ PG, and poly-unsaturated DLoPG $(18: 2$ cis $\Delta^{9,12}$ ) (structures on Figure $\mathrm{S} 1$ ). The resulting photocrosslinked species with intact phospholipids are heteroadducts known to be challenging to observe in MS. To allow a better MS ionisation/detection yield, phospholipids saponification was performed on the photoadducts before affinity purification of the biotinylated species on streptavidin-coated magnetic beads and MALDI-TOF (Figure 2) or ESI-Orbitrap (Figure S4) analysis, thus leading to photoadducts composed of the peptide covalently linked to a simple fatty acid. ${ }^{13}$ This step is crucial for the robustness of the setup. Measuring the mass shift $(\Delta \mathrm{m})$ between the ion signal of the non-crosslinked peptide (Pen, or $\left.(\mathrm{R} / \mathrm{W})_{9}\right)$ and the photoadducts allows identification of the fatty acid covalently linked to the peptide. Expected adducts, corresponding to a C-C bond formation (molecules $\mathbf{2}$ and $\mathbf{3}$ ) between the photoreactive Pen and the different intact fatty acids are referred to in the manuscript as: Pen+myristic acid (MA; 14:0), Pen+oleic acid $\left(\mathrm{OA} ; 18: 1\right.$ cis $\Delta^{9}$ ) Pen+linoleic acid (LoA; 18:2 cis $\Delta^{9,12}$ ) or in SI Pen+palmitic acid (PA; 16:0), Pen+elaidic acid (EA; 18:1 trans $\Delta^{9}$ ) (Figure S5). All results obtained with Pen are presented in the main text. Results with (R/W) 9 are all shown in SI (Figures S6, S7). Both peptides gave comparable results.

Optimisation of the photocrosslinking protocol (see SI) led to good quality ion signals corresponding to the expected peptide/fatty acid photoadducts as shown in the MALDI-MS spectra acquired using 2,5 Dihydroxybenzoic acid (2,5-DHB) as the matrix (Figure 2). 2,5-DHB is used since this is the matrix of choice to ionize lipids ${ }^{16,17}$ and peptide-lipid adducts. ${ }^{13}$

High S/N ratio MALDI MS spectra allowed the unambiguous detection of low intense intermediate ions in the region between the $\mathrm{m} / \mathrm{z}$ of the non-crosslinked Pen and the $\mathrm{m} / \mathrm{z}$ of the intact photoadduct. These intermediate ions (highlighted in the dashed box and zoom in Figure 2) are only observed after photoirradiation (+hv spectra). Using an ESI ion source, these intermediate ions are even more intense (Figure S4). Therefore, these intermediate ions must be treated as products of the UV-induced secondary reactions, and not occurring during the UV MALDI irradiation.

We will first describe in details the Bzp photoreactivity in a membrane with a focus on the isotopic distributions for the Pen + intact fatty acid photoadducts, and the reactions leading to the intermediate ions observed in the MS spectra.

\section{UV-induced redox reactions involving Bzp}

The isotopic distribution of the Pen-MA photoadduct obtained with MALDI (Figure 2) and ESI (Figure S4) ion sources differs from the one predicted by the IsoPro software for the pure compound. The experimental isotopic distribution of Pen-MA photoadduct (theoretical first isotope at $\mathrm{m} / \mathrm{z}$ 3384) contains a contribution at $-2 \mathrm{u}(\mathrm{m} / \mathrm{z} 3382)$ as indicated in Figure 2. On Figure $3 \mathrm{~A}$, left panel, we superimposed the experimental isotopic distribution of the Pen-MA ion signal (blue dots), the theoretical distribution (black triangles) and simulation of a pattern resulting of two species with a $-2 \mathrm{u}$ difference (orange diamonds). Isotopic pattern simulation showed that the species at $-2 u$ contributes for $75 \%$ to the experimental signal. This pattern is observed for PenMA in MALDI and ESI MS spectra showing that this photoreactivity of the Bzp is not due to the UV laser $(\lambda=355 \mathrm{~nm})$ during the MALDI ionisation process, but occurs in solution during the photolabelling step. This is also observed for the PenPA adduct (Figure S5) but not observed with Pen-OA (Figure 3A, middle panel), Pen-EA (Figure S5) or Pen-LoA adducts (Figure $3 \mathrm{~A}$, right panel).

We explain this shift towards lower $\mathrm{m} / \mathrm{z}$ by specific redox photoreactivity of the Bzp towards saturated lipids. As described in solution by Breslow, ${ }^{2,3}$ Bzp can induce the formation of a double bond on a rigid and constrained substrate. In Figure 3B, we propose such UV-induced mechanism occurring on the fatty acid chain of a lipid in a vesicle composed of saturated phospholipids that provides the constrained environment. In the system described by Breslow, rigidity is brought by the constrained molecular scaffold whereas in our system, rigidity is imposed by the saturated membrane. This UV-induced reaction consisting in the abstraction of two radical hydrogens (formal loss of $\mathrm{H}_{2}$ ) (molecule 4, Figure 1) competes with the photocrosslinking reaction leading 
to the $\mathrm{C}-\mathrm{C}$ bond formation between Bzp and a fatty acid (molecule 2, Figure 1). Considering the kinetics of $\mathrm{H}^{*}$ abstraction by Bzp on secondary, allylic and doubly allylic hydrogens, ${ }^{18}$ competitive formation of the double bond will be favoured on saturated lipids (Figure S8). The combination of the lower rigidity of an unsaturated $v s$ saturated lipid membrane and the favourable kinetics of the $\mathrm{C}-\mathrm{C}$ bond formation on allylic or doubly allylic positions explains why the $-2 \mathrm{u}$ contribution is not observed on photoadducts obtained with DOPG, trans-C18:1 and DLoPG.

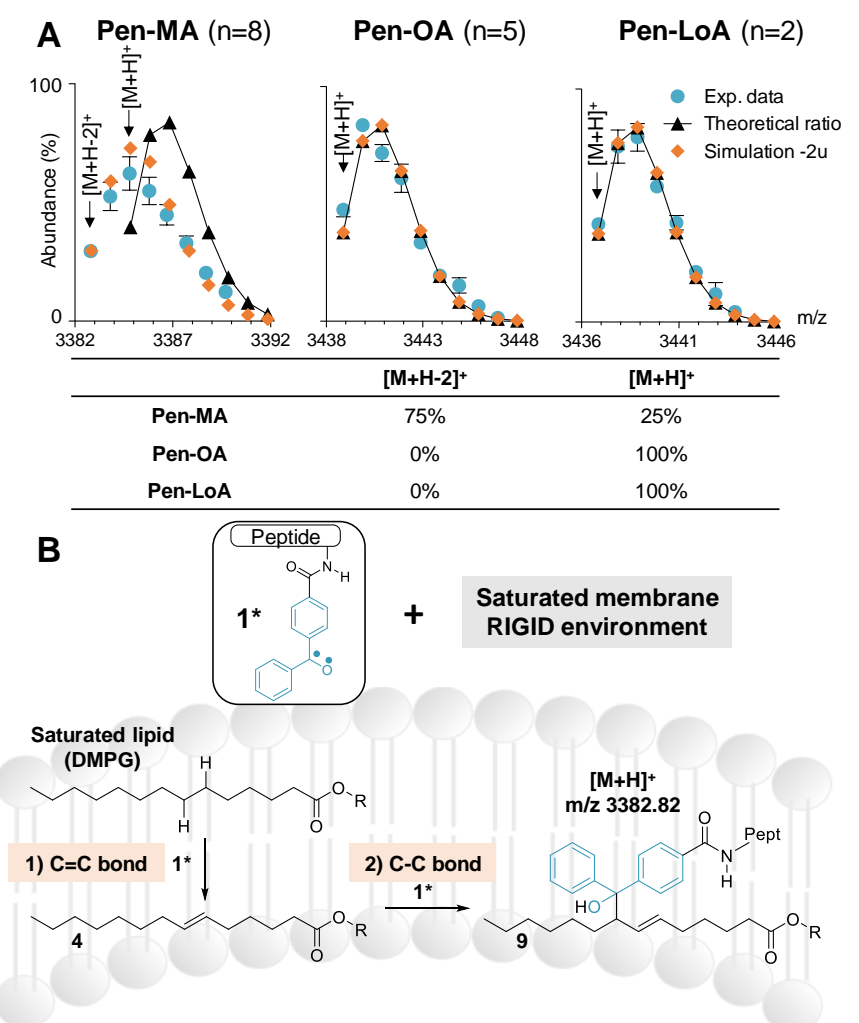

Figure 3. A) Isotopic ratios of the Pen-lipid photoadducts with MA (DMPG), OA (DOPG) and LoA (DLoPG) (error bars correspond to standard deviation). B) UV-induced photoredox activity of the Bzp leading to double bond formation in a constrained DMPG MLV.

Once the double bond is formed on a saturated fatty acid (molecule 4, Figure 3B), the photocrosslinking reaction is favoured on the newly-modified fatty acid (molecule 9, Figure $3 \mathrm{~B})$. This consecutive reaction involves the presence of a second Pen with activated Bzp (molecule 1*) in the close vicinity of the new unsaturation, which is highly probable in the conditions used for the photocrosslinking reaction (lipid:peptide ratio 10:1). Furthermore, since the membrane possibly becomes more fluid around the defect caused by the double bond, insertion of the Pen and the probe will be facilitated in this area. Indeed, Pen is known to interact more favourably with lipids in fluid phase. ${ }^{19}$

\section{UV-induced covalent bond formation and rupture}

Intact photoadducts were characterised by fragmentation using high energy CID (MALDI-TOF/TOF) and post-source decay (PSD) gave the same information (Figures S10, S12). A favoured fragmentation of the covalent bond formed between Pen and the fatty acids is obtained, as previously observed for peptide-peptide photoadducts $^{20}$, showing that they all contain the intact Pen sequence. Neutral loss leading to Pen fragment ion (m/z 3156) corresponds to the loss of the lipid part covalently bound to Pen, confirming that the proton is located on the peptide. The fragmentation of the Pen-LoA entire photoadduct leads to a highly intense fragment ion peak at the $\mathrm{m} / \mathrm{z}$ of Pen, showing that this newly-formed bond is unstable and can be easily broken during CID, or even afterwards in the flight tube (after reacceleration of the fragment ions) as shown by the broad fragmentation signal just next to the parent ion. Its fragility is probably increased compared to MA and OA due to the presence of multiple double bonds in the LoA, stabilising the fragment ion resulting from the loss of the fatty acid (tertiary radical ion). This is in agreement with the relative intensities of the photoadducts and the Pen ion signals (Figure 2), which are inversely proportional to the relative stability of the radical resulting from the newly-formed C-C bond fragmentation.

Further MS/MS data analysis revealed that photoadducts mask two different photoinduced reactivities of the Bzp towards lipids: $\mathrm{C}-\mathrm{C}$ bond formation (molecules $\mathbf{2}$ and $\mathbf{3}$ ) and [2+2] cycloaddition leading to oxetane formation (Paternò-Büchi reaction, $\mathrm{P}-\mathrm{B}$ ) on unsaturated OA, LoA (molecule 5 and Figure S13) and oxidised MA. ${ }^{21-23}$ These two pathways lead to products with the same elemental composition, which makes them undistinguishable at the MS level. MS/MS spectra of the intact photoadducts therefore contain fragment ions originating from both species.

Bzp is a P-B reagent used for characterisation and quantification of unsaturated lipids in solution or in the gas-phase by MS/MS. ${ }^{21,24,25}$ Giving energy to the system results in a retro Paternò-Büchi (retro P-B) reaction leading to ions characteristics of the position of the double bond. ${ }^{21,26,27}$ The corresponding $\alpha$ and $\beta$ ions observed in our study (schemed in Figure 4) are directly observed in MALDI and ESI MS spectra. They are present in MS/MS with very low intensity and with consecutive fragments $\gamma$ and $\delta$ (Figure 4 ) in the low $\mathrm{m} / \mathrm{z}$ region (Figure 5) proving that both $\mathrm{C}-\mathrm{C}$ bond and oxetane ring formation coexist. The $\mathrm{m} / \mathrm{z}$ values of the $\alpha, \beta, \gamma$ and $\delta$ ions observed are listed in Table S1. Two intermediate ions observed in Figure 2 with DOPG at m/z 3266.71 and 3296.71 (Figure 2) indeed match masses of the expected retro $\mathrm{P}-\mathrm{B}$ products $\beta$ and $\alpha$, respectively (Table $\mathrm{S} 1$ ).

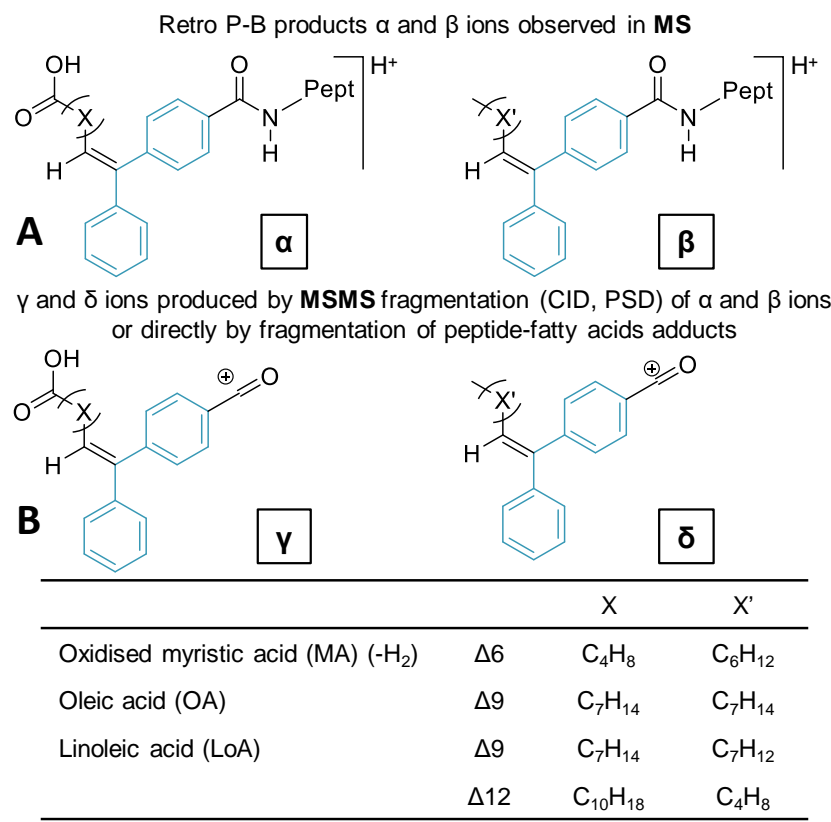

Figure 4. Retro P-B products $\alpha, \beta, \gamma, \delta$ obtained in MS and/or MSMS when photoreactive Pen is crosslinked to unsaturated lipids. A) $\alpha, \beta$ ions observed in MS, B) $\gamma, \delta$ ions observed in MSMS. 
Oxetane ring opening through retro $\mathrm{P}-\mathrm{B}$ can be done by low collision energy in MS/MS, by heating ${ }^{24,25,28}$ or acidic treatment since monosubstituted oxetanes are known to be unstable at $\mathrm{pH}$ $1 .{ }^{29}$ Our experiment requires heating at $45^{\circ} \mathrm{C}$ for $1 \mathrm{~h}$ during the photolabelling step in solution, and acidic $\mathrm{pH}(1-2)$ in the 2,5DHB MALDI matrix or in the ESI solvent (formic acid $0.1 \%$ ). There is also steric hindrance due to the presence of Pen on the Bzp. Therefore, both retro P-B $\alpha$ and $\beta$ products can be observed at the MS level which is a real asset in our analytical approach.

Further fragmentation happens when additional energy is applied to the retro P-B intermediate $\alpha$ and $\beta$ ions or P-B regioisomer products leading to diagnostic $\gamma$ and $\delta$ fragment ions (Figure 4 and Figure 5, m/z at 319, 349 for the Pen-OA adduct, numbered peaks 5 ' and 7 , respectively and $\mathrm{m} / \mathrm{z}$ at $277,317,349$ for the Pen-LoA adduct, numbered peaks 2, 5 and 7, respectively). This offers structural information about the photocrosslinked $\mathrm{C}=\mathrm{C}$ bond of the unsaturated lipids. Retro P-B fragments $\gamma$ and $\delta$ at $\mathrm{m} / \mathrm{z}$ 349 and 319/317 show comparable relative intensities. This is in agreement with the P-B regioselectivity, which is mainly linked to steric hindrance of the substituents on the olefin and is not an issue in our case. ${ }^{30}$ Both attacks of the carbonyl of the Bzp are equiprobable on the fatty acid double bond. Other ions are reproducibly observed with similar relative intensities, in the low mass range in all MS/MS spectra. They correspond to Pen b-ions as well as ions at $\mathrm{m} / \mathrm{z} 209,259,292$, the structures of which are schemed in Figure 5 (ions 1, 2, 4) according to mechanisms published in the literature. ${ }^{31}$ A structure is proposed for the ion at $\mathrm{m} / \mathrm{z} 331$ (ion 6) observed with DOPG.

We did not identify retro P-B products $\alpha$ and $\beta$ for DLoPG on MS spectra probably due to the fragility of the oxetane ring which leads back to the original reactants: Pen and LoA (Figure S13).

More interestingly, P-B reaction can happen as a second step reaction on DMPG, after formation of the double bond as described previously. In fact, both $\mathrm{C}-\mathrm{C}$ bond and oxetane rings (Figure 1) can be generated on the newly-formed unsaturated lipid $(\mathrm{m} / \mathrm{z} 3382$, corresponding to the $-2 \mathrm{u}$ shift of the ion at 3384, Figure 2). The intermediate ion observed at $\mathrm{m} / \mathrm{z} 3254.74$ on MS spectrum of Pen+DMPG+hv (Figure 2) matches the mass of the retro P-B product $\alpha$ (Table S1). This is particularly interesting as the retro P-B product allows MS characterisation of the position of the newly formed double bond on MA as being on C6-C7 (total mass of the retro P-B product ion corresponding to the mass of Pen $+98 u$ ), without further MS/MS fragmentation. In the case of saturated phospholipids, all positions having an equivalent reactivity towards $\mathrm{H}^{*}$ abstraction by Bzp, the positioning of the double bond gives a direct indication of the insertion depth of Pen in the membrane. No P-B fragments $\gamma$ and $\delta$ are observed in MS/MS with DMPG, probably due to the relatively low quantity of photoadduct at $\mathrm{m} / \mathrm{z} 3382$, which impairs acquisition of MS/MS spectra with high $\mathrm{S} / \mathrm{N}$.

\section{Bzp-induced photosensitisation}

In our experiments with DMPG or DOPG, we could reproducibly detect low intense covalent photoadducts corresponding to Pen covalently linked to two fatty acids in the higher $\mathrm{m} / \mathrm{z}$ region. Corresponding MALDI-TOF spectra are presented in Figure S14. One Bzp moiety cannot form two covalent bonds with two distinct fatty acids, the presence of such adducts can thus only be explained by dimerisation of fatty acids due to Bzp-induced photosensitisation of an unsaturated fatty acid (Figure 1, molecules $6^{*}$ and 7) two distinct activated Bzp moeities $\left(1^{*}\right)$ are involved: a crosslinker and a sensitiser.

Bzp-induced dimerisation through photosensitisation has been well described for biomolecular interactions with DNA. Bzp photoinduced reactions (photooxidation, P-B or retro P-B reactions, C-C bond formation, dimerisation) often competes (Figure 1). ${ }^{1}$ A study by Liu et al. on Bzp and pyrimidine bases revealed that the proportion of $\mathrm{P}-\mathrm{B}$ reaction compared to dimerisation via photosensitisation, depends on the relative triplet energy $\left(E_{T}\right)$ levels of both entities. ${ }^{32}$ When the $E_{T}$ values of the Bzp and the pyrimidine are close, the two reactions occur concurrently. In our case, the product of dimerisation via Bzp photosensitisation are always detected as low intensity ions compared to other Bzp photoinduced products, indicating that this pathway is probably not energetically favoured, provided protonation occurring on the Pen moiety and therefore ionisation yields comparable for all types of photoproducts.

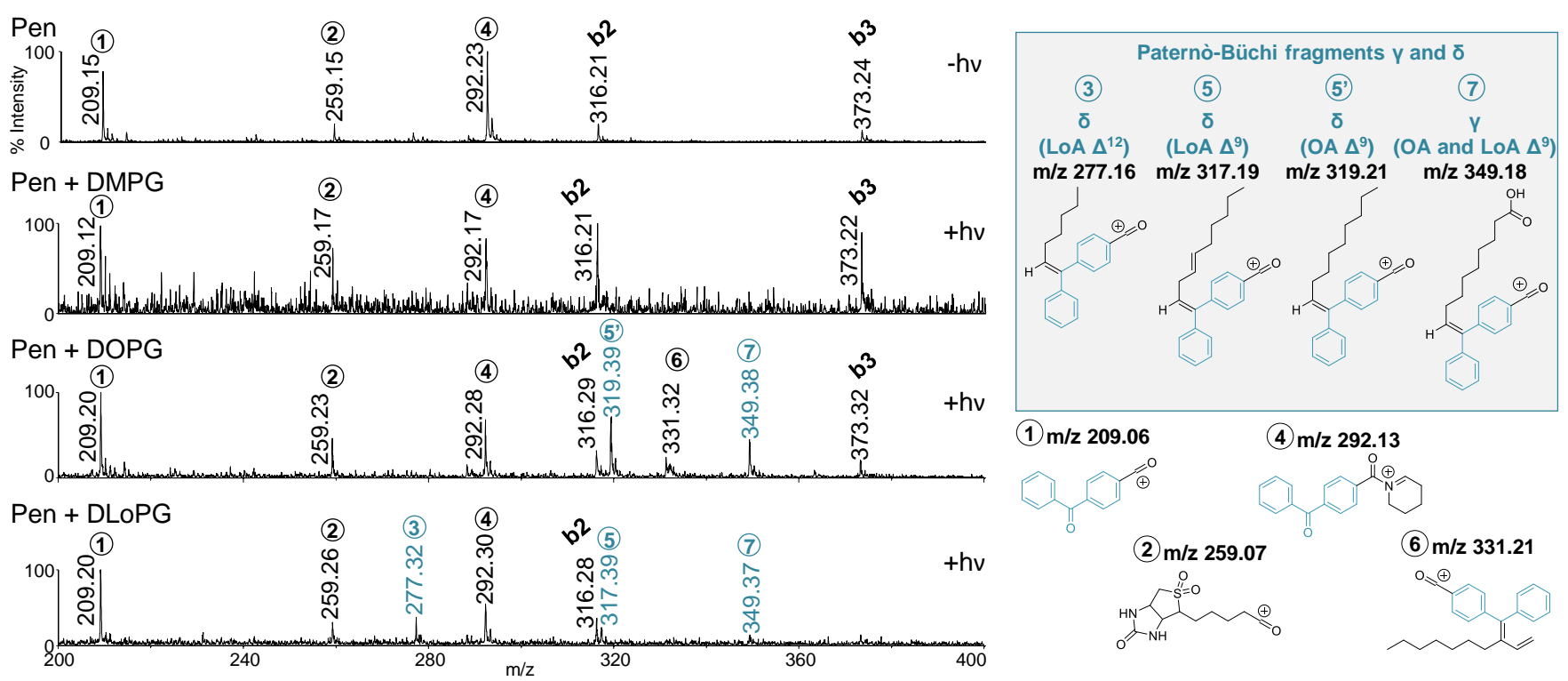

Figure 5. Low mass range $200<\mathrm{m} / \mathrm{z}<400$ MALDI-TOF/TOF spectra of the Pen+fatty acids intact photoadducts precursor ions. Control Pen without irradiation $(-h v)$, Pen + DMPG $+h v$, Pen+DOPG $+h v$, Pen+DLoPG $+h v$. Structures of ions are numbered from 1 to 7 and P-B fragments are highlighted in the grey box. 
Very interestingly, we observe fatty acid dimerisation with DOPG, but also DMPG which confirms the photooxidation (loss of $\mathrm{H}_{2}$ ) of MA. Without this newly-formed unsaturation, photosensitised dimerisation of MA would not occur.

Finally, it is described in the literature that UV irradiation of Bzp in solution in isopropanol leads to homodimerisation of ketyl radicals to form benzopinacol, but no such dimerisation is reported for protein-Bzp ${ }^{1}$ or peptide-Bzp ${ }^{20}$. Similarly, in our experiments in lipid membranes, we observed no homodimerisation (Figure 1, molecule 8) of Pen or (R/W) $)_{9}$ either in the presence of liposomes or by irradiation of the control Pen or $(\mathrm{R} / \mathrm{W})_{9}$ alone.

\section{Radical oxygen species-induced oxidation reactions}

Apart from P-B products $\alpha$ and $\beta$, the intermediate ions region of MS spectra (Figure 2) contains species at $+140.1 /+138.1$, $+154.1 /+152.1$ and $+168.1 /+166.1$ compared to the $\mathrm{m} / \mathrm{z}$ of the non-crosslinked Pen for unsaturated DOPG and DLoPG. It is known that double bonds of (poly)unsaturated fatty acids can be cleaved during UV irradiation by reaction with radical oxygen species (ROS) ${ }^{33}$ (Figure S15). Theoretically, we could observe these ions with DMPG after double bond formation, but none are observed, probably due to the higher acyl packing preventing penetration of water soluble radicals and oxidation. ${ }^{33}$ These oxidation ions thus reflect membrane fluidity around the CPP.

\section{New insights in Pen preference for fluid-phase bilayers}

After identifying all side-products and reactions, as described above, we go further on the characterisation of CPP/lipids interaction in a membrane bilayer. Since MALDI ionisation of the photoadducts only relies on the peptide moiety, the intensity of the ion species likely reflects the relative proportion between the photoadducts and the peptide in the MLVs. ${ }^{13}$ Therefore, we assume that the relative intensities between Pen and photoadducts are proportional to the photolabeling yields, which are very different depending on the PG acyl chain (Figure 2 and S5). These photolabeling yields can be ranked as follows: OA > MA PA EA $>$ LoA (or cis 18:1 > 14:0 16:0 trans 18:1 > cis 18:2). Pen-OA photoadduct shows the highest relative intensity compared to Pen. These results cannot be explained with the $\mathrm{H}^{*}$ abstraction kinetics that are more rapid for doubly allylic over allylic and secondary hydrogens (kinetics given in Figure S8). The cis 18:1 unsaturation (OA) is indeed more crosslinked than the trans 18:1 one (EA). Trans fatty acids adopt a linear configuration similar to that of saturated fatty acid, while cis fatty acids have a bent configuration. Trans fatty acids are more packed than cis ones. This suggests that Pen shows preferential interactions with OA lipid chains found in fluid phase. ${ }^{19}$ It is therefore not surprising that Pen-MA, Pen-PA and Pen-EA photoadducts show comparable relative intensities compared to Pen. These acyl chains being saturated or transunsaturated are indeed found in more packed membrane domains (see main transition temperatures given in the Experimental section). In addition, although DLoPG should give the fastest kinetics thanks to the doubly allylic $\mathrm{H}^{\cdot}$ abstraction ${ }^{18}$ (Figure S8), the corresponding photoadduct (Pen-LoA) has the lowest intensity signal relative to the one of Pen (Figure 2). This is likely due to the low stability of the formed adduct compared to other acyl chains, as supported by tandem mass spectrometry (MS/MS; Figure S10) data that have been discussed above.

Altogether, these first results with PG acyl chains only clearly establish that $\mathrm{H}^{\cdot}$ abstraction kinetics do not dominate photocrosslink reactions of PG MLVs with Pen and that other key parameters such as the membrane fluidity has to be taken into account to understand Pen photoadducts.

\section{Analytical workflow and application of the methodology}

On Figure 6, we propose a simple analytical workflow for the comprehensive interpretation of MS spectra of Bzp-based $\mathrm{CPP} /$ lipid photocrosslinking experiments taking into account all the secondary reactions we described above.

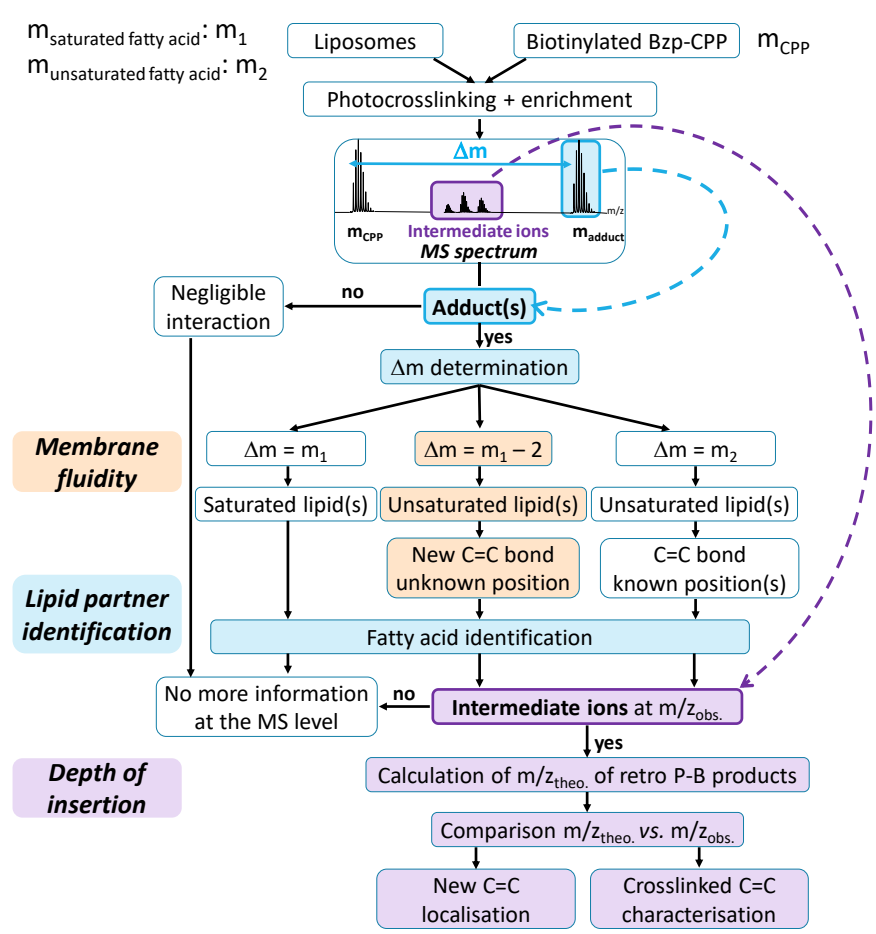

Figure 6. Analytical workflow for the comprehensive interpretation of the photocrosslinking MS spectra.

Information on the interaction can be obtained at three levels. Identification of the CPP/fatty acid adduct(s) tells about lipid recognition preference by the CPP (Figure 6, teal boxes). Analysis of the isotope distribution of the adduct(s) peak(s) and in particular the presence of the first isotope at m-2 compared to the expected $\mathrm{m} / \mathrm{z}$, gives information about the fluidity of the membrane in the vicinity of the Bzp (Figure 6, orange boxes). Finally, careful identification of potential intermediate ions gives an indication on the depth of insertion of the Bzp probe in the membrane, as it allows determination of the position of the photocrosslinked $\mathrm{C}=\mathrm{C}$ bond as well as the position of any potentially newly formed $\mathrm{C}=\mathrm{C}$ bond on the crosslinked fatty acid (Figure 6, lilac boxes).

To illustrate this wokflow, we studied the interaction of ApaPen (an analogue of Pen with an additional Apa spacer between the $\operatorname{Biot}\left(\mathrm{O}_{2}\right)$ tag and the peptide sequence) with binary membranes composed of DMPG/Chol or DOPG/Chol $(75: 25 \mathrm{~mol} / \mathrm{mol})$. It is indeed very interesting to study the interaction of CPPs with Chol -containing membranes as it was previously showed that Chol is involved in the internalisation mechanisms of tryptophan-rich CPPs such as Pen or $(\mathrm{R} / \mathrm{W})_{9} \cdot{ }^{34} \mathrm{Chol}$ is known to condense and rigidify lipid bilayers containing phospholipids with unsaturated fatty acyl chains such as DOPG. In contrast, it has been found that Chol has preferential interactions with saturated lipids, ${ }^{35}$ and that it fluidizes bilayers of di-saturated phospholipids. ${ }^{36}$ The obtained MS spectra are presented on Figure 7 (DMPG/Chol) and Figure S16 (DOPG/Chol). For DMPG/Chol, two adducts are observed 
(m/z at 3481 and 3641), corresponding to ApaPen+MA and ApaPen+Chol respectively. This shows that Chol molecules are present in the close vicinity of the CPPs bound to the membrane, likely because the peptide prefers the more fluid phase of the DMPG/Chol mixture. On the other hand, with DOPG/Chol, no ApaPen+Chol could be observed. This suggests that the interaction between ApaPen and DOPG is tighter than with DMPG or that ApaPen tends to exclude Chol from its immediate environment in the more rigid DOPG/Chol bilayer, to stay in a more fluid phase.

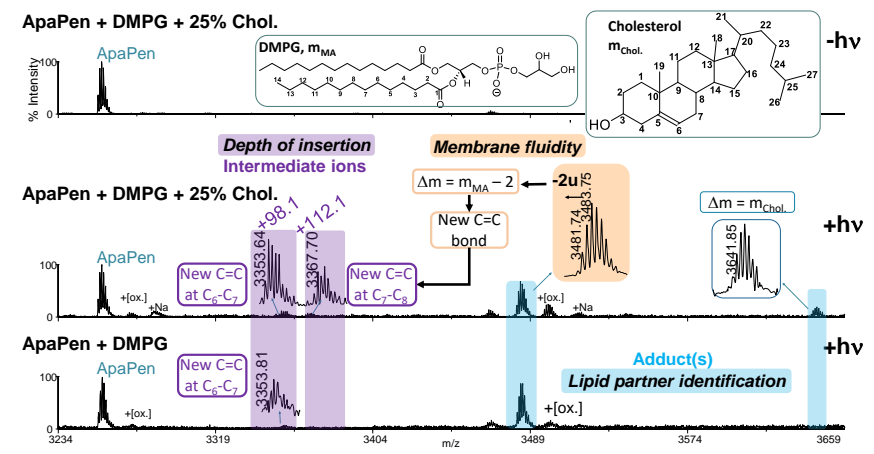

Figure 7. MALDI-TOF spectra (positive ions, reflector mode) of the Bzp-induced photocrosslinking between ApaPen and binary MLVs. Top to bottom: Control ApaPen+DMPG+25\% Chol without irradiation (-hv), ApaPen+DMPG+hv and ApaPen+DMPG $+25 \%$ Chol+hv. Ions at 3353 and 3367 are $\alpha$ retro $\mathrm{P}-\mathrm{B}$ product ions with $\mathrm{X}=$ $\left(\mathrm{CH}_{2}\right)_{4}$ and $\mathrm{X}=\left(\mathrm{CH}_{2}\right)_{5}$, respectively. $\mathrm{m}_{\mathrm{MA}}=228, \mathrm{~m}_{\mathrm{Chol}}=386$.

When looking at isotope distribution of the adduct signals, the Chol adduct is at the expected $\mathrm{m} / \mathrm{z}$ and the MA adduct still contains a contribution at $-2 \mathrm{u}$. With DOPG, the OA adduct is at the expected $\mathrm{m} / \mathrm{z}$, showing that the presence of $25 \%$ of Chol in the membrane does not rigidify the membrane enough to allow the formation of an additional $\mathrm{C}=\mathrm{C}$ bond on $\mathrm{DOPG}$ or that the peptide recruits DOPG (and excludes $\mathrm{Chol}$ ) to stay in a more fluid environment. Finally, when looking at the intermediate ions, two distinct retro P-B products are clearly identified with DMPG in the presence of $25 \% \mathrm{Chol}(\mathrm{m} / \mathrm{z}$ at 3353 and 3367) whereas only one is detected in the absence of $\mathrm{Chol}(\mathrm{m} / \mathrm{z}$ at 3353$)$. In the latter case, the intermediate ion corresponds to the formation of the $\mathrm{C}=\mathrm{C}$ bond between $\mathrm{C} 6$ and $\mathrm{C} 7$ as described above. The C6-C7 position suggests a rather shallow insertion of ApaPen in the lipid bilayer, which is consistent with what is known about Pen interactions with membranes. Pen carries many cationic residues and their deep insertion in the membrane would come with a very high energetic cost. Furthermore, Pen carries two W residues, which are known to position themselves at water/lipid interfaces rather than inserting deeply within membranes. ${ }^{37} \mathrm{We}$ previously showed that Bzp-modified Pen has little effect on the fluidity and organisation of a DMPG bilayer as probed by DSC. ${ }^{14}$ In the presence of Chol, the additional intermediate ion corresponds to the formation of the $\mathrm{C}=\mathrm{C}$ bond between $\mathrm{C} 7$ and $\mathrm{C} 8$, showing heterogeneous depth of insertion, with some Bzp moieties reaching deeper in the hydrophobic core of the membrane. This clearly shows that the presence of $\mathrm{Chol}$ has a disordering effect on the DMPG membrane and allows deeper insertion of the CPP. Because retro P-B on Chol would only lead to ring opening with no mass modification compared to the Chol adduct, no information on the position of the formed covalent bond can be obtained. However, the most likely positions are the $\mathrm{C} 4$ and $\mathrm{C} 7$ allylic carbons on Chol. Labelling on $\mathrm{C} 7$ would be consistent with the position of Chol in a phospholipid membrane. ${ }^{38}$

\section{CONCLUSION}

In this study, we give a full description of Bzp photoreactivity within a lipid bilayer and illustrate how it can be exploited to study the interactions of two CPPs, Pen and (R/W) ${ }_{9}$ with lipid interaction partners at the molecular level. The studied reactions encompass classical $\mathrm{C}-\mathrm{C}$ bond formation, oxidation of the fatty acid chains via double bond $\mathrm{C}=\mathrm{C}$ formation, Paternò-Büchi reaction as well as Bzp-induced photosensitisation leading to unsaturated lipids dimerisation. Additionally, oxidation reactions leading to double bond cleavage can be observed on unsaturated lipids. The occurrence of consecutive reactions requiring two UV-activated Bzp suggests that CPPs accumulate in the same region and are close to each other. Mapping of these secondary reactions is important, for at least three reasons, which we summarised on an analytical workflow showing how to extract the maximum information out of a simple MS spectrum (Figure 6). First, ignoring the photoredox reactions involving Bzp could lead to false interpretations or photoadduct identification, especially when working with binary mixtures containing saturated and unsaturated lipids with the same chain length (Figure 6, teal boxes). Second, careful analysis of isotopic distribution can give information on membrane fluidity in the immediate environment of the CPP (Figure 6, orange boxes). Additionally, presence of ions resulting from ROS-induced oxidation of the CPP/lipids photoadducts gives an indication on membrane fluidity which influences penetration of water soluble radicals and oxidation. Finally, analysis of the retro P-B products allows finer characterisation of the CPP/lipid interaction at the atomic level without using MS/MS, giving information on the depth of insertion of the CPP in the membrane (Figure 6, lilac boxes). We illustrate this by analysing the interaction of Penetratin with membranes composed of a binary mixture of PG and Chol.

Knowing how to extract the information at the MS level will be an asset for studying interaction of CPPs with complex biological membranes composed of multiple lipids. This is a great advantage when analysing sub-picomolar amounts of a given species knowing that MS/MS requires more material than MS experiments.

Finally, this is the first report of Paternò-Büchi reactivity and Bzp-induced photosensitisation in a Bzp-based lipid photocrosslinking setup. Such reactivity was reported to occur with DNA, ${ }^{28,39}$ suggesting the universality of this phenomenon, which should never be overlooked when studying biomolecular interactions with Bzp-based photocrosslinking. In addition, our study shed new light on the insertion depth of CPPs with regard to membrane fluidity, and brings evidence for the relevance of such approach using liposomes as an intermediate before going to cells.

\section{Supporting information Available}

Detailed experimental procedures and $(\mathrm{R} / \mathrm{W})_{9}$ results are given in the Supporting Information.

\section{Conflicts of interest}

There are no conflicts to declare.

\section{ACKNOWLEDGEMENTS}

We thank the MS and Proteomics Platform IBPS, SU for equipment and assistance and the MS platform IPCM, SU. We thank Y. Gimbert, G. Poli and A. Pradal IPCM, SU for fruitful discussions. LB has a PhD grant from the French Ministère de l'Enseignement Supérieur, de la Recherche et de l'Innovation (M.E.S.R.I.). 


\section{REFERENCES}

(1) Dormán, G.; Nakamura, H.; Pulsipher, A.; Prestwich, G. D. The Life of Pi Star: Exploring the Exciting and Forbidden Worlds of the Benzophenone Photophore. Chem. Rev. 2016, 116 (24), 15284 15398.

(2) Breslow, R.; Baldwin, S.; Rechtner, T.; Kalicky, P.; Liu, S.; Washburnle, W. Remote Oxidation. J. Am. Chem. Soc. 1973, 95 (3), 3251-3262.

(3) Breslow, R. Artificial Enzymes; Breslow, R., Ed.; John Wiley \& Sons, 2006.

(4) Fréneau, M.; Hoffmann, N. The Paternò-Büchi ReactionMechanisms and Application to Organic Synthesis. J. Photochem. Photobiol. C Photochem. Rev. 2017, 33, 83-108.

(5) Walrant, A.; Cardon, S.; Burlina, F.; Sagan, S. Membrane Crossing and Membranotropic Activity of Cell-Penetrating Peptides: Dangerous Liaisons? Acc. Chem. Res. 2017, 50 (12), 2968-2975.

(6) Haberkant, P.; Raijmakers, R.; Wildwater, M.; Sachsenheimer, T.; Brügger, B.; Maeda, K.; Houweling, M.; Gavin, A. C.; Schultz, C.; Van Meer, G.; et al. In Vivo Profiling and Visualization of Cellular Protein-Lipid Interactions Using Bifunctional Fatty Acids. Angew. Chemie - Int. Ed. 2013, 52 (14), 4033-4038.

(7) Xia, Y.; Peng, L. Photoactivatable Lipid Probes for Studying Biomembranes by Photoaffinity Labeling. Chem. Rev. 2013, 113 (10), 7880-7929.

(8) Gubbens, J.; Ruijter, E.; de Fays, L. E. V.; Damen, J. M. A.; de Kruijff, B.; Slijper, M.; Rijkers, D. T. S.; Liskamp, R. M. J.; de Kroon, A. I. P. M. Photocrosslinking and Click Chemistry Enable the Specific Detection of Proteins Interacting with Phospholipids at the Membrane Interface. Chem. Biol. 2009, 16 (1), 3-14.

(9) Kölbel, K.; Ihling, C. H.; Sinz, A. Analysis of Peptide Secondary Structures by Photoactivatable Amino Acid Analogues. Angew. Chemie - Int. Ed. 2012, 51 (50), 12602-12605.

(10) Peng, T.; Yuan, X.; Hang, H. C. Turning the Spotlight on ProteinLipid Interactions in Cells. Curr. Opin. Chem. Biol. 2014, 21, 144 153.

(11) Kramer, K.; Sachsenberg, T.; Beckmann, B. M.; Qamar, S.; Boon, K. L.; Hentze, M. W.; Kohlbacher, O.; Urlaub, H. Photo-CrossLinking and High-Resolution Mass Spectrometry for Assignment of RNA-Binding Sites in RNA-Binding Proteins. Nat. Methods 2014, 11 (10), 1064-1070.

(12) Kawaguchi, Y.; Takeuchi, T.; Kuwata, K.; Chiba, J.; Hatanaka, Y.; Nakase, I.; Futaki, S. Syndecan-4 Is a Receptor for ClathrinMediated Endocytosis of Arginine-Rich Cell-Penetrating Peptides. Bioconjug. Chem. 2016, 27 (4), 1119-1130.

(13) Jiao, C.-Y.; Sachon, E.; Alves, I. D.; Chassaing, G.; Bolbach, G.; Sagan, S. Exploiting Benzophenone Photoreactivity to Probe the Phospholipid Environment and Insertion Depth of the CellPenetrating Peptide Penetratin in Model Membranes. Angew. Chemie Int. Ed. 2017, 56 (28), 8226-8230.

(14) Jiao, C. Y.; Alves, I. D.; Point, V.; Lavielle, S.; Sagan, S.; Chassaing, G. Comparing Lipid Photo-Cross-Linking Efficacy of Penetratin Analogues Bearing Three Different Photoprobes: Dithienyl Ketone, Benzophenone, and Trifluoromethylaryldiazirine. Bioconjug. Chem. 2010, 21 (2), 352-359.

(15) Delaroche, D.; Aussedat, B.; Aubry, S.; Chassaing, G.; Burlina, F.; Clodic, G.; Bolbach, G.; Lavielle, S.; Sagan, S. Tracking a New CellPenetrating (W/R) Nonapeptide, through an Enzyme-Stable Mass Spectrometry Reporter Tag. Anal. Chem. 2007, 79 (5), 1932-1938.

(16) Schiller, J.; Arnhold, J.; Benard, S.; Mu, M.; Reichl, S.; Arnold, K Lipid Analysis by Matrix-Assisted Laser Desorption and Ionization Mass Spectrometry: A Methodological Approach. Anal. Biochem. 1999, 267, 46-56.

(17) Schiller, J.; Süß, R.; Fuchs, B.; Müller, M.; Petkovic, M.; Zschörnig, O.; Waschipky, H. The Suitability of Different DHB Isomers as Matrices for the MALDI-TOF MS Analysis of Phospholipids: Which Isomer for What Purpose? Eur. Biophys. J. 2007, 36 (4-5), 517-527.

(18) Lala, A. K.; Kumar, E. R. Orientation of the Benzophenone Group at Various Depths in Bilayers. J. Am. Chem. Soc. 1993, 115 (10), 3982-3988.

(19) Joanne, P.; Galanth, C.; Goasdoué, N.; Nicolas, P.; Sagan, S.; Lavielle, S.; Chassaing, G.; El Amri, C.; Alves, I. D. Lipid Reorganization Induced by Membrane-Active Peptides Probed Using Differential Scanning Calorimetry. Biochim. Biophys. Acta -
Biomembr. 2009, 1788 (9), 1772-1781.

Clavier, S.; Bolbach, G.; Sachon, E. Photocross-Linked PeptideProtein Complexes Analysis: A Comparative Study of CID and ETD Fragmentation Modes. J. Am. Soc. Mass Spectrom. 2015, 26 (6), 1014-1026.

(21) Xu, T.; Pi, Z.; Song, F.; Liu, S.; Liu, Z. Benzophenone Used as the Photochemical Reagent for Pinpointing $\mathrm{C}=\mathrm{C}$ Locations in Unsaturated Lipids through Shotgun and Liquid ChromatographyMass Spectrometry Approaches. Anal. Chim. Acta 2018, 1028, 3244.

(22) Bednařík, A.; Bölsker, S.; Soltwisch, J.; Dreisewerd, K. An OnTissue Paternò-Büchi Reaction for Localization of Carbon-Carbon Double Bonds in Phospholipids and Glycolipids by Matrix-Assisted Laser-Desorption-Ionization Mass-Spectrometry Imaging. Angew. Chemie - Int. Ed. 2018, 57 (37), 12092-12096.

(23) Esch, P.; Heiles, S. Charging and Charge Switching of Unsaturated Lipids and Apolar Compounds Using Paternò-Büchi Reactions. $J$. Am. Soc. Mass Spectrom. 2018, 29 (10), 1971-1980.

(24) Ma, X.; Xia, Y. Pinpointing Double Bonds in Lipids by PaternòBüchi Reactions and Mass Spectrometry. Angew. Chemie Int. Ed. 2014, 53 (10), 2592-2596.

(25) Stinson, C. A.; Xia, Y. A Method of Coupling the Paternò-Büchi Reaction with Direct Infusion ESI-MS/MS for Locating the CC Bond in Glycerophospholipids. Analyst 2016, 141 (12), 3696-3704. (26) Jeck, V.; Korf, A.; Vosse, C.; Hayen, H. Localization of DoubleBond Positions in Lipids by Tandem Mass Spectrometry Succeeding High-Performance Liquid Chromatography with Post-Column Derivatization. Rapid Commun. Mass Spectrom. 2018, No. May, 19.

(27) Wäldchen, F.; Becher, S.; Esch, P.; Kompauer, M.; Heiles, S. Selective Phosphatidylcholine Double Bond Fragmentation and Localisation Using Paternò-Büchi Reactions and Ultraviolet Photodissociation. Analyst 2017, 142 (24), 4744-4755.

(28) Nakatani, K.; Yoshida, T.; Saito, I. Photochemistry of Benzophenone Immobilized in a Major Groove of DNA: Formation of Thermally Reversible Interstrand Cross-Link. J. Am. Chem. Soc. 2002, 124 (10), 2118-2119.

(29) Burkhard, J. A.; Wuitschik, G.; Rogers-Evans, M.; Müller, K.; Carreira, E. M. Minireviews Oxetanes as Versatile Elements in Drug Discovery and Synthesis. Angew. Chemie Int. Ed. 2010, 49, 90529067.

(30) Kumarasamy, E.; Raghunathan, R.; Kandappa, S. K.; Sreenithya, A.; Jockusch, S.; Sunoj, R. B.; Sivaguru, J. Transposed Paternò-Büchi Reaction. J. Am. Chem. Soc. 2017, 139 (2), 655-662.

(31) Atik, A. E.; Hernandez, O.; Maître, P.; Yalcin, T. Specific Rearrangement Reactions of Acetylated Lysine Containing Peptide Bn (N=4-7) Ion Series. J. Mass Spectrom. 2014, 49 (12), 1290-1297. (32) Liu, X. L.; Wang, J. B.; Tong, Y.; Song, Q. H. Regioselectivity and Competition of the Paternò-Büchi Reaction and Triplet-Triplet Energy Transfer between Triplet Benzophenones and Pyrimidines: Control by Triplet Energy Levels. Chem. - A Eur. J. 2013, 19 (39), 13216-13223.

(33) Reis, A.; Spickett, C. M. Biochimica et Biophysica Acta Chemistry of Phospholipid Oxidation. Biochim. Biophys. Acta - Biomembr. 2012, 1818 (10), 2374-2387.

(34) Bechara, C.; Pallerla, M.; Burlina, F.; Illien, F.; Cribier, S.; Sagan, S. Massive Glycosaminoglycan-Dependent Entry of Trp-Containing Cell-Penetrating Peptides Induced by Exogenous Sphingomyelinase or Cholesterol Depletion. Cell. Mol. Life Sci. 2015, 72 (4), 809-820. (35) Radhakrishnan, A.; McConnell, H. Condensed Complexes in Vesicles Containing Cholesterol and Phospholipids. Proc. Natl. Acad. Sci. 2005, 102 (36), 12662-12666.

(36) Maxfield, F. R.; van Meer, G. Cholesterol, the Central Lipid of Mammalian Cells. Curr. Opin. Cell Biol. 2010, 22 (4), 422-429.

(37) Killian, J. A.; Heijne, G. Von. How Proteins Adapt to a MembraneWater Interface. 2000, 0004 (September), 429-434.

(38) Ermilova, I.; Lyubartsev, A. P. Cholesterol in Phospholipid Bilayers: Positions and Orientations inside Membranes with Different Unsaturation Degrees. Soft Matter 2019, 15 (1), 78-93.

(39) Verga, D.; Hamon, F.; Poyer, F.; Bombard, S.; Teulade-Fichou, M. P. Photo-Cross-Linking Probes for Trapping G-Quadruplex DNA. Angew. Chemie - Int. Ed. 2014, 53 (4), 994-998.

For Table of Contents Only 


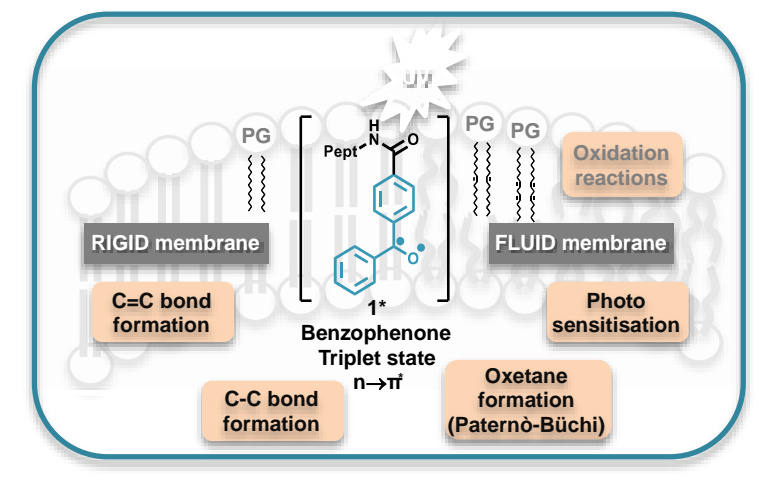

Complete analytical characterisation at the MS level of the intermediate ions issued from $\mathrm{P}-\mathrm{B}$ or retro $\mathrm{P}-\mathrm{B}$ reactions provides new insights for the interpretation of MS spectra with regard to information on the depth of insertion of peptides in membranes made of saturated lipids. 